Orange Journal / Volumen 3 Número 5/ Enero - junio 2021

DOI: https://doi.org/10.46502/issn.2710-995X/2021.5.02

Mesa Pujals, A.A., Hernández Cortés, K.S., \& Montoya Pedrón, A. (2021). Manejo de overlays para la volumetría cerebral. Orange Journal, 3(5), 10-15. https://doi.org/10.46502/issn.2710-995X/2021.5.02

\title{
Manejo de overlays para la volumetría cerebral
}

\section{Overlays management for brain volumetric}

Recibido: 10 de Julio de 2021 Aceptado: 30 de agosto de 2021

\author{
Escrito por: \\ Adrian Alberto Mesa Pujals ${ }^{3}$ \\ https://orcid.org/0000-0003-0643-5315 \\ Katherine Susana Hernández Cortés ${ }^{4}$ \\ https://orcid.org/0000-0001-9293-9450 \\ Arquímedes Montoya Pedrón ${ }^{5}$ \\ https://orcid.org/0000-0001-9415-4585
}

\section{Resumen}

El análisis cuantitativo de los datos de imágenes clínicas es un área activa de investigación prometedora para la medicina de precisión, la evaluación temprana de la respuesta al tratamiento y la caracterización objetiva de la enfermedad. La interoperabilidad, el intercambio de datos y la capacidad de extraer los mismos son de importancia creciente, dado el crecimiento explosivo en el número de métodos de análisis cuantitativos que se proponen. La forma estandarizada de almacenar esta información en la imagen es mediante la utilización del módulo DICOM "Overlays". Muchos fabricantes de sistemas imagenológicos de visualización producen implementaciones no estándar de los overlays (e.g. xml) para almacenar las anotaciones o no consideran la utilización (lectura, escritura) de múltiples capas, según se especifica en DICOM. Esta problemática también está presente en los PACS desplegados en el Sistema de Salud Cubano. Imagis 3.0 es una herramienta que soluciona las limitaciones actuales, permitiendo la conversión de los datos almacenados en formatos de investigación de uso común en la representación DICOM estándar. Al igual que sus versiones precedentes, ofrece al usuario un conjunto de herramientas que facilitan e incrementan la eficiencia del sistema de salud. Esta investigación tiene como objetivo presentar un módulo de gestión de overlays. Se revisaron las publicaciones, tanto en PubMed como el estado del arte de los resultados. Se utilizaron las imágenes de tomografía axial computarizada de cráneo en pacientes cuya cifra ascendió a 120. Se presentaron varios componentes de software especializados en: 1) codificar segmentaciones de imágenes cerebrales como overlays de forma estandarizada; 2) almacenar hasta 16 capas independientes según el estándar DICOM. Se definieron múltiples regiones de interés independientes sobre una misma imagen y se conservó el orden de realización con fines docentes. Se concluye que este módulo puede ser considerado una herramienta para realizar evaluaciones cuantitativas de las neuroimágenes.

Palabras claves: DICOM, Neuroimágenes, Overlays, PACS, Volumetría cerebral.

\begin{abstract}
For the neuroscience community, quantitative analysis of clinical imaging data is a promising active area of research for precision medicine, early assessment of response to treatment, and objective characterization of disease. Interoperability, data exchange and the ability to extract information from neuroimaging are becoming increasingly important given the growth of quantitative analysis methods that are proposed in this regard. The

\footnotetext{
${ }^{3}$ Ingeniero en Ciencias Informáticas del Centro de Biofísica Médica. Especialista B de Ciencias Informático. Universidad de Oriente, Santiago de Cuba. Cuba.

${ }^{4}$ Especialista de Primer Grado en Anatomía Humana. Máster en Medicina Bioenergética y Natural. Profesor Asistente de la Universidad de Ciencias Médicas de Santiago de Cuba. Metodóloga de la Dirección de Ciencia e Innovación Tecnológica de la Universidad de Ciencias Médicas de Santiago de Cuba. Grupo de Investigación: Neurociencias. Santiago de Cuba. Cuba.

${ }^{5}$ Doctor en Ciencias Médicas. Especialista de Primer y Segundo Grado en Neurofisiología Clínica. Profesor Titular e Investigador Titular. Jefe del servicio de Neurofisiología del Hospital General Docente Dr. Juan Bruno Zayas Alfonso. Santiago de Cuba. Presidente de la comisión de grado de Ciencias Médicas. Jefe del grupo de Neurociencias. Santiago de Cuba. Cuba.
} 


\section{ORANGE JOURNAL}

persistence of segmentations as regions of interest in medical images makes possible to communicate relevant diagnostics information between specialists in neuroscience. The standard way to store this information inside a medical image file is through the use of overlays as defined in the DICOM standard.

Many PACS viewer manufacturers either develop non-standard overlays implementations (e.g. using xml) to store annotations or do not consider the reading or writing of overlay planes, in accordance with DICOM specifications. This problem is present in PACS solutions deployed in Cuban Healthcare System as well. Imagis 3.0 is a tool that allows the conversion of a user's segmentation information in commonly used research formats into standard DICOM representation. Like its previous versions, it offers the user a set of tools that facilitate the work of specialists and doctors and increase the efficiency of the health system. Like its previous versions, it offers the user a set of tools that facilitate the work of specialists and doctors and increase the efficiency of the health system. This paper presents an overlays management module. Many publications are cited in this survey, including benchmark datasets and state of the art results. For the present perspective study, axial computerized tomography (CT) of head from more than 120 patients. In particular, we present some software components specialized in: 1) coding segmentations of regions of interest as overlays according to DICOM standard; 2) storing until 16 independent layers in accordance with DICOM standard. This makes possible to define multiples ROIs in one image, and preserves the annotation creation order for teaching or research purposes. Moreover, this module is considered a tool to make cuantitative evaluations.

Key Words: DICOM, Neuroimages, Overlays, PACS, brain volumetric.

\section{Introducción}

El análisis cuantitativo de los datos contenida en las imágenes médicas es una cuestión importante en todos los sistemas imagenológicos de visualización. El mayor avance científico que pueden registrar las técnicas de neuroimágenes es que no requieren cirugías o procedimientos en los que se ponga en riesgo la vida humana, sino que al ser llamadas no invasivas permiten el estudio del cerebro y, más aún, del cerebro con vida y en pleno funcionamiento. Se aplican con gran frecuencia en las enfermedades neurodegenerativas y en las demencias, sirviendo para establecer principios diagnósticos mucho más claros y precisos, tanto en la identificación de patrones de enfermedad, como en daños estructurales y funcionales provocados por toda la amplia gama de enfermedades degenerativas. En los últimos años, debido a la necesidad de realizar el diagnóstico precoz, el esfuerzo se ha centrado en el desarrollo de técnicas de neuroimágenes que permitan lograr la detección de enfermedad en etapas preclínicas, antes que el daño cerebral irreversible se produzca. (del Pilar, Rueda \& Enríquez, 2018). Los métodos de segmentación automática y la identificación de regiones de interés (ROI por sus siglas en inglés), anotaciones y mediciones dentro de una imagen, constituye una práctica común para reflejar información asociada al diagnóstico. (Yu \& Wang, 2010)

Debido a la tendencia creciente hacia la conservación e intercambio de información dentro y entre instituciones médicas, el uso de overlays representa un aspecto clave para los sistemas de almacenamiento, búsqueda y recuperación de imágenes conocidos como PACS (Pictures Archiving and Communication Systems). (Yu \& Wang, 2010) (Li, 2003)

En Cuba existe cierta experiencia con esta tecnología relacionada con los servicios médicos, ejemplo de ello es el desarrollo y la implementación del archivo de imágenes y sistemas de comunicación (PACS) y lanzó la base para el desarrollo de PACS: Soluciones llamadas iMagis que son hoy en día la más difundida en Cuba. IMagis es un conjunto de soluciones PACS desarrolladas bajo la Plataforma Linux, utilizando herramientas gratuitas, que se compone de un servidor DICOM y un sistema de visualización. Al igual que todas las soluciones PACS, ofrece servicios de almacenamiento, indexación, búsqueda y entrega de imágenes, desde modernos equipos de imágenes médicas y estaciones de trabajo (Daudinot, \& Miller, 2016).

Especialistas médicos como: radiólogos, neurocirujanos, ortopédicos entre otros, utilizan las regiones de interés, mediciones, anotaciones, etc., para la planificación de procedimientos quirúrgicos y de radioterapia. Estas anotaciones son muy importantes para las actividades docentes, investigativas y en la práctica clínica. De aquí la necesidad de disponer de mecanismos eficientes, flexibles y estandarizados para conservar esta información. (del Pilar Rueda \& Enríquez, 2018). 
En los sistemas PACS el manejo de las anotaciones, mediciones y ROIs se realiza generalmente a través del objeto DICOM (Digital Imaging and Communication in Medicine) conocido como Overlays (DICOM Standard Commite, ACR Nema, 2014).

Muchos sistemas PACS utilizados en el Sistema Nacional de Salud (SNS) e.g., Efilm v3.4, Weasis, carecen o proveen una implementación limitada de los overlays definidas en DICOM (Mesa, Pardiñas \& Kindelán, 2017).

En este trabajo se propone una solución que resuelve las limitaciones enunciadas. En particular, se presenta un mecanismo para almacenar overlays de segmentaciones de regiones de interés conforme a la definición especificada en DICOM PS3.3 apéndice C9 (Li, Wilson, Wong, \& Xthona, 2003). Este enfoque permite almacenar hasta 16 overlays asociados a una imagen. Esto resulta útil para conservar la secuencia de creación de segmentaciones de regiones de interés con fines docentes, clínicos y de investigación.

\section{Marco Teórico}

Según el estándar DICOM, un overlay es una máscara binaria que describe gráficos, textos y regiones de interés asociadas a la imagen. La máscara se obtiene a partir de la segmentación de regiones de interés realizadas en la imagen (Moeller, \& Reif, 2000).

Nos referimos a segmentación de regiones de interés cuando un volumen de imagen generalmente representa un gran número de imágenes que se oscurecen entre sí. Para mostrar uno en particular, tenemos que decidir qué partes del volumen queremos usar o ignorar. El primer paso es dividir el volumen de la imagen en diferentes regiones que son homogéneas con respecto a algunos criterios formales y que corresponden a objetos reales (anatómicos). Este proceso se llama segmentación (Moeller, \& Reif, 2000) (Spalletta, Piras, \& Gili,2018).

La selección de la /las regiones de interés no es más que la selección de que parte o estructura se va a emplear en el estudio y cual se va a ignorar, esto depende del objetivo del investigador (Moeller, \& Reif, 2000) (Spalletta, Piras, \& Gili,2018).

La matriz de píxeles de cada imagen obtenida, es considerada como una máscara binaria, donde cada valor distinto de 0 (i.e., negro) se hace igual a 1.

La codificación del objeto overlay se realiza según la definición especificada en DICOM PS3.3 apéndice C9 (ver Tabla 1).

Tabla 1.

Atributos del Módulo Overlay

\begin{tabular}{|l|l|}
\hline Nombre del Atributo & Descripción del Atributo \\
\hline Overlay Rows & Número de filas en el Overlay \\
\hline Overlay Columns & Número de columnas en el Overlay \\
\hline Overlay Type & Indica si el overlay representa una región de interés o un gráfico. \\
& $\begin{array}{l}\text { Valores: } \\
\text { G Gráfico. } \\
\text { R ROI. }\end{array}$ \\
\hline Overlay Origin & $\begin{array}{l}\text { Localización del primer punto del overlay con respecto a los pixel en la } \\
\text { imagen, tomando como fila/columna. }\end{array}$ \\
\hline Overlay Data & Matriz de pixel del overlay. \\
\hline
\end{tabular}

\section{Metodología}

Se desarrolló un estudio retrospectivo, transversal, descriptivo y observacional en el servicio de imagenología del Hospital Saturnino Lora de Santiago de Cuba entre enero a junio del año 2021. 


\section{ORANGE JOURNAL}

El universo de estudio estuvo conformado por 2400 imágenes de 120 pacientes de ambos sexos con edades comprendidas entre 35-74 años de edad, se manejó la aceptación de participar en la investigación, los cuales tenían indicaciones previas de TC de cráneo. Se seleccionó este rango de edades por considerarse que alrededor de los 40 años comienza la etapa de neurodegeneración (del Pilar, Rueda \& Enríquez, 2018).

Se incluyeron pacientes con diagnósticos de enfermedades neurológicas y psiquiátricas, enfermedades degenerativas como la ataxia espino cerebelosa, trastornos neurocognitivos (para lo cual se les aplicó el mini examen del estado mental (MEEM) estandarizado y aprobado para la población cubana), trastornos esquizofrénicos Como criterio de exclusión se consideró el embarazo y traumatismos cráneo encefálicos que comprometiesen la anatomía de la región.

Se preservó el cumplimiento ético de las investigaciones biomédicas promulgados en la declaración de Helsinki y el informe de Belmont.

El escáner CT utilizado en este estudio fue el SIEMENS, mono corte. Los factores de exposición seleccionados para la tomografía computarizada fue 80-120 kvp y 250-450 más, y el índice de ruido fue de 3. Todas las tomografías computarizadas se realizaron en modo axial con algoritmo interactivo de reconstrucción.

El paciente fue colocado en la mesa PET / CT y la cabeza fue centralizada y soportada para mantener una posición correcta y para evitar desenfoque de la imagen. Se tomó una imagen de exploración lateral para confirmar posicionamiento del paciente. Se dibujó una línea orbito-meatal y una línea en un se dibujó un ángulo de 15 - 20 grados y $1 \mathrm{~cm}$ por encima, representando la sección tomográfica más baja, que pasó a través de la base de cráneo.

El tiempo total de la TC fue de 50-60 segundos. Cada paciente tiene entre 18 a 22 cortes con un grosor de $5 \mathrm{~mm}$ en este estudio. El tamaño de la matriz de cada segmento es de 512 x 512 píxeles y el tamaño de píxel es de $0,426 \mathrm{~mm}$ con un nivel de gris de 16 bits. Todo el procesamiento y la medición de imágenes axiales de TC se realizaron en la estación de trabajo, por los integrantes del equipo de investigación. Las imágenes fueron evaluadas por un especialista de segundo grado en Imagenología.

Para la construcción de la solución, destinada a la creación de overlays estandarizados según DICOM, se utilizaron las siguientes herramientas:

- Biblioteca que posibilita el acceso a información DICOM a través de VTK: vtk-dicom.

- Biblioteca para la visualización y procesamiento de datos e imágenes: VTK (Visualization Toolkit).

- Biblioteca para la segmentación y registros: ITK (Insight Segmentation and Registration Toolkit)

- $\quad$ Lenguaje de programación $\mathrm{C}++$.

Para la validación de la solución desarrollada se emplearon:

- $\quad$ Sistemas de software de referencia: Efilm 3.4 y Weasis 2.0.3.

- En la validación de la lectura de overlays se utilizaron:

- 50 imágenes DICOM con overlays producidas por escáner CT SIEMENS, así como los sistemas Efilm v3.4, Weasis 2.0.3 y nuestra solución para la lectura de imágenes con overlays.

- Respecto a la validación de la escritura de overlays se utilizaron:

- 50 imágenes médicas de cabeza sin overlays de modalidades CT provenientes de terceros. Los overlays son creados por la solución propuesta de segmentación semi-automatizada y visualizados como los sistemas de referencia Efilm v3.4 y Weasis 2.0.3.

El método propuesto para la creación de overlays a partir de anotaciones presentes en la imagen, está estructurado en dos fases: segmentación y codificación del overlay. La fase de segmentación se ejecuta en 
el proceso de extracción, clasificación y cuantificación de rasgos anatómicos. Al almacenar la imagen se invoca la fase de codificación y se termina el proceso de conformación del overlay.

\section{Resultados y discusión}

Se obtuvo un Sistema para la Gestión de Overlays $\left(\mathrm{SGO}_{\mathrm{v} 2}\right)$ en su segunda versión conforme al estándar DICOM compuesto por: un conjunto de algoritmos para la segmentación de las regiones de interés (ROI) y una biblioteca de clases para la codificación y lectura de varias capas de overlays en la imagen médica. La tabla 2 muestra los resultados correspondientes a la validación de la lectura de overlays. Puede observarse que el Efilm v3.4 y Weasis 2.0.3 visualizaron todas las imágenes con sus overlays aunque no pueden manipular las capas, ni mostrar informacion cuantitativa. Similarmente, el SGO visualiza todas las imágenes con overlays, además permite manipular todas las capas de overlay y mostrar informacion cuantitativa adicional.

Tabla 2.

Resultados de validación de lectura.

\begin{tabular}{|l|l|l|}
\hline Visor PACS & Imágenes Visualizadas & Manejo de Overlays \\
\hline SGO & $100 \%$ & Visualiza/oculta Overlays \\
\hline Efilm v3.4 & $100 \%$ & Visualiza Overlays \\
\hline Weasis 2.0 .3 & $100 \%$ & Visualiza Overlays \\
\hline
\end{tabular}

En la tabla 3 se reflejan los resultados correspondientes a la validación de escritura de overlays generados por SGO. Los datos muestran que los overlays generados por el SGO fueron visualizados por Efilm v3.4 y Weasis 2.0.3. Esto demuestra que el SGO genera overlays conformes al estándar DICOM 3.0.

Tabla 3.

Resultados de escritura.

\begin{tabular}{|l|l|}
\hline Visor PACS & Imágenes visualizadas \\
\hline Efilm v3.4 & $100 \%$ \\
\hline Weasis 2.0 .3 & $100 \%$ \\
\hline
\end{tabular}

Muchos sistemas PACS utilizados en el Sistema Nacional de Salud (SNS) e.g., Efilm v3.4, Weasis, carecen o proveen una implementación limitada de los overlays definidas en DICOM, por esta razón la interoperabilidad, el intercambio de datos y la capacidad de extraer los mismos es limitada.

En el sistema Weasis, el manejo de Overlays, se realiza a través del formato de ficheros *.xml, donde se almacena el tipo (e.g., ángulos, distancias, etc.) y las coordenadas de las anotaciones. Esto reduce la portabilidad del método porque los overlays de las imágenes no pueden ser visualizados en otros visores. Por otro lado, el Efilm en su versión 3.4, posibilita la visualización de overlays pero no provee ningún mecanismo para almacenarlas de forma estandarizada.

\section{Conclusiones}

- Se obtuvo una herramienta, para el almacenamiento de overlay en imágenes médicas DICOM compatibles.

- La herramienta facilita la interoperabilidad, el intercambio de datos y la capacidad de extraer los mismos.

- La herramienta posibilita la navegación de overlay por capas, evitando que se superpongan unas con otras.

- Las imágenes con overlays, generadas por la herramienta son correctamente codificadas según el estándar DICOM.

- La herramienta puede incorporarse sobre terminales de trabajo con fines docentes, clínicos y de investigación.

- Se comprobó que la solución propuesta supera otros sistemas desplegados en el SNS. 


\section{ORANGE JOURNAL}

\section{Referentes Bibliográficas}

Daudinot, López, M., \&Miller, Clemente, R. (2016). Una solución pacs cubana bajo software libre que sirve de plataforma a especializaciones médicas. Revista cubana de informática médica, 8(2), 186-196.

del Pilar, Rueda., \& Enríquez, LF. (2018). Una revisión de técnicas básicas de neuroimagen para el diagnóstico de enfermedades neurodegenerativas. Biosalud, 17(2), 59-90.

DICOM Standard Commite, ACR Nema. (2014). PS3.6 DICOM PS3.6 2014c - Data Dictionary.

DICOM Standard Commite, ACR Nema. (2014). PS3.3 DICOM PS3.3 2014c - Explanatory Information. DICOM Standard.

DICOM Standard Commite, ACR Nema. (2014). PS3.5 DICOM PS3.5 2014c - Data Structures and Encoding.

Li, M., Wilson, D., Wong, M., \& Xthona, A. (2003). The evolution of display technologies in PACS applications. Computerized medical imaging and graphics, 27(2-3), 175-184.

Liu, Y., \& Wang, J. (2010). PACS and digital medicine: essential principles and modern practice. CRC Press.

Malaterre, M. (2016). GDCM (Grassroots DICOM). A DICOM library.

Mesa, AA., Pardiñas, NH., \& Kindelán, R. (2017). Manejo de overlays para escenarios Clínicos y Docentes. Memorias de la Convención Internacional de Ciencias Técnicas (CD-ROM). Ruta: IPDF\Archivo: Memorias.pdf ISSN: 2218-6719

Moeller, TB., \& Reif, E. (2000). Normal Findings in CT and MRI. Thieme New York, 1-26.

Spalletta, G., Piras, F., \& Gili, T. (2018). Brain Morphometry. Neuromethods, 136, 165-170. 